\title{
AMENDMENTS
}

\section{Author Correction: The copy number variation landscape of congenital anomalies of the kidney and urinary tract}

\author{
Miguel Verbitsky DD, Rik Westland, Alejandra Perez, Krzysztof Kiryluk, Qingxue Liu, Priya Krithivasan, Adele Mitrotti, \\ David A. Fasel (D), Ekaterina Batourina, Matthew G. Sampson (DD, Monica Bodria, Max Werth, Charlly Kao, \\ Jeremiah Martino, Valentina P. Capone, Asaf Vivante, Shirlee Shril, Byum Hee Kil, Maddalena Marasa (D), Jun Y. Zhang, \\ Young-Ji Na, Tze Y. Lim, Dina Ahram, Patricia L. Weng, Erin L. Heinzen (D, Alba Carrea, Giorgio Piaggio, Loreto Gesualdo, \\ Valeria Manca, Giuseppe Masnata, Maddalena Gigante (D), Daniele Cusi, Claudia Izzi, Francesco Scolari, \\ Joanna A. E. van Wijk, Marijan Saraga, Domenico Santoro, Giovanni Conti, Pasquale Zamboli, Hope White, Dorota Drozdz, \\ Katarzyna Zachwieja, Monika Miklaszewska, Marcin Tkaczyk, Daria Tomczyk, Anna Krakowska, Przemyslaw Sikora, \\ Tomasz Jarmoliński, Maria K. Borszewska-Kornacka, Robert Pawluch, Maria Szczepanska, Piotr Adamczyk, \\ Malgorzata Mizerska-Wasiak, Grazyna Krzemien, Agnieszka Szmigielska, Marcin Zaniew, Mark G. Dobson, \\ John M. Darlow, Prem Puri, David E. Barton D, Susan L. Furth, Bradley A. Warady, Zoran Gucev, Vladimir J. Lozanovski, \\ Velibor Tasic, Isabella Pisani, Landino Allegri, Lida M. Rodas, Josep M. Campistol, Cécile Jeanpierre, Shumyle Alam, \\ Pasquale Casale, Craig S. Wong, Fangming Lin, Débora M. Miranda, Eduardo A. Oliveira, Ana Cristina Simoes-e-Silva, \\ Jonathan M. Barasch, Brynn Levy, Nan Wu DiD, Friedhelm Hildebrandt ID, Gian Marco Ghiggeri, Anna Latos-Bielenska, \\ Anna Materna-Kiryluk, Feng Zhang, Hakon Hakonarson, Virginia E. Papaioannou (D), Cathy L. Mendelsohn (D), \\ Ali G. Gharavi (D) and Simone Sanna-Cherchi iD
}

Correction to: Nature Genetics https://doi.org/10.1038/s41588-018-0281-y, published online 21 December 2018.

In the version of this article initially published, affiliation 38 incorrectly read "ICNU-Nephrology and Urology Department, Barcelona, Spain"; "Renal Division, Hospital Clinic, IDIBAPS, University of Barcelona, Barcelona, Spain" is the correct affiliation. The error has been corrected in the HTML and PDF versions of the article.

\section{Author Correction: Repurposing large health insurance claims data to estimate genetic and environmental contributions in 560 phenotypes}

\author{
Chirag M. Lakhani, Braden T. Tierney (D), Arjun K. Manrai, Jian Yang (D), Peter M. Visscher (D) and Chirag J. Patel (D) \\ Correction to: Nature Genetics https://doi.org/10.1038/s41588-018-0313-7, published online 14 January 2019.
}

In the version of Fig. $4 \mathrm{~b}$ initially published, there was a calculation error in the estimates of shared environmental variance $\left(c^{2}\right)$ for MaTCH functional domains. For all MaTCH functional domains except the 'all traits' functional domain, the estimate of $c^{2}$ was calculated with monozygotic twin correlation $\left(r_{\mathrm{MZ}}\right)$ and dizygotic twin correlation $\left(r_{\mathrm{DZ}}\right)$ for each functional domain provided by the MaTCH website (http://match.ctglab.nl/). The $c^{2}$ value should have been estimated as $c^{2}=2 r_{\mathrm{DZ}}-r_{\mathrm{MZ}}$ but, owing to a coding error, was erroneously estimated as $c^{2}=2 r_{\mathrm{DZ}}-r_{\mathrm{DZ}}$. The $c^{2}$ estimate for the 'all traits' functional domain was correct in the version of the article initially published, and therefore no conclusions are affected; however, the contribution of $c^{2}$ among MaTCH functional domains is decreased. The authors thank G. Gibson and M. Nordborg for pointing out the error.

To correct this error, Fig. 4 has been revised to include corrected $c^{2}$ estimates in the data in panel $\mathrm{b}$ as well as to include the numbers of phenotypes in both the CaTCH and MaTCH functional domains in the $y$ axes of panels a and $\mathrm{b}$. The number of phenotypes for each MaTCH functional domain in Fig. 4 is based on the number of phenotypes for which $h^{2}$ and $c^{2}$ were estimated with twin correlation $\left(r_{\mathrm{MZ}}\right.$ and $\left.r_{\mathrm{DZ}}\right)$ taken from the MaTCH website. The total numbers of phenotypes within each MaTCH functional domain where $h^{2} / c^{2}$ were estimated with either twin correlation or variance component models (ACE) and can be found in Supplementary Table 1. The legend of Fig. 4 has been revised to include descriptions of the red and blue values and a description of the numbers of phenotypes in the $y$ axes in panels a and b. In the Results section, the description of Fig. $4 \mathrm{~b}$ reading "For $c^{2}$, the 95\% CI from CaTCH estimates overlapped with the 95\% CI from the MaTCH estimates for only the infection domain (Fig. $4 \mathrm{~b}$ )" has been changed to "For $c^{2}$, the $95 \%$ CI from $\mathrm{CaTCH}$ estimates overlapped with the $95 \% \mathrm{CI}$ from the $\mathrm{MaTCH}$ estimates for 11 out of 21 functional domains, namely cardiovascular, dermatological, endocrine, gastrointestinal, hematological, immunological, infection, metabolic, psychiatric, reproduction, and skeletal functional domains (Fig. 4b)." 
In the version of the article initially published, the erroneous term 'depravity index', appearing throughout the main text, Fig. 1, Supplementary Fig. 10 and the Supplementary Note, should have read 'deprivation index'.

The errors have been corrected in the HTML and PDF versions of the article.
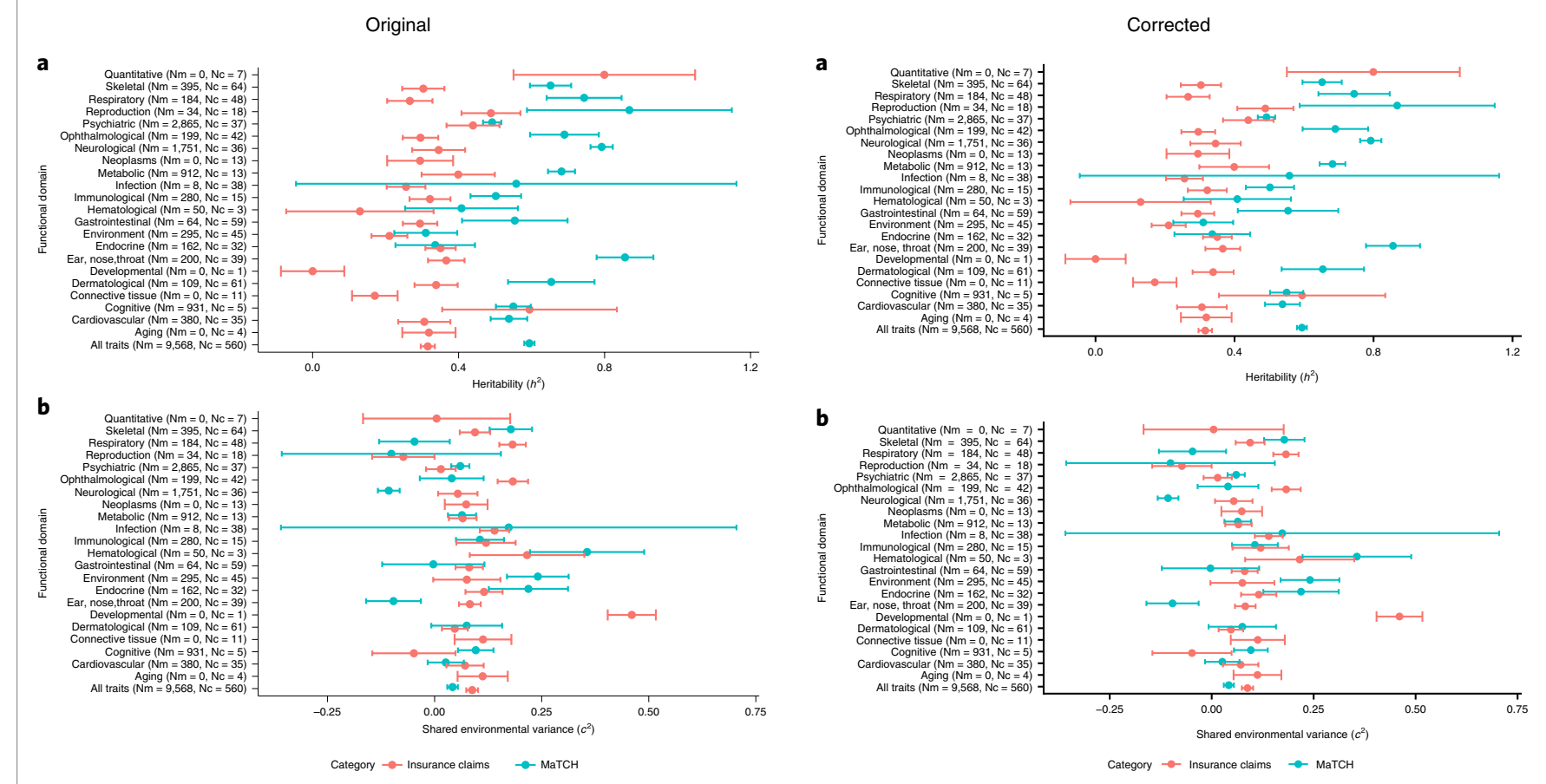

Fig. 4 | Original and corrected.

Published online: 27 February 2019

https://doi.org/10.1038/s41588-019-0377-z

\section{Author Correction: Origin and evolution of the octoploid strawberry genome}

Patrick P. Edger (D), Thomas J. Poorten, Robert VanBuren, Michael A. Hardigan, Marivi Colle, Michael R. McKain (D), Ronald D. Smith (D), Scott J. Teresi, Andrew D. L. Nelson (D), Ching Man Wai, Elizabeth I. Alger, Kevin A. Bird (D), Alan E. Yocca, Nathan Pumplin, Shujun Ou Did, Gil Ben-Zvi (D), Avital Brodt, Kobi Baruch, Thomas Swale, Lily Shiue, Charlotte B. Acharya, Glenn S. Cole, Jeffrey P. Mower, Kevin L. Childs, Ning Jiang, Eric Lyons, Michael Freeling (iD, Joshua R. Puzey and Steven J. Knapp (D)

Correction to: Nature Genetics https://doi.org/10.1038/s41588-019-0356-4, published online 25 February 2019.

In the version of this article originally published, author Joshua R. Puzey was incorrectly listed as having affiliation 7 (School of Plant Sciences, University of Arizona, Tucson, AZ, USA); affiliation 6 (Department of Biology, College of William and Mary, Williamsburg, VA, USA) is the correct affiliation. The error has been corrected in the HTML and PDF versions of the article.

Published online: 6 March 2019

https://doi.org/10.1038/s41588-019-0380-4 